\title{
Cutaneous Rosai-Dorfman Disease Confused with Vascular Mass
}

\author{
Kwang Rae Kang, \\ Sung Won Jung, \\ Sung Hoon Koh \\ Department of Plastic and Reconstructive \\ Surgery, Hallym University Sacred Heart \\ Hospital, Hallym University Medical Center, \\ Anyang, Korea
}

No potential conflict of interest relevant to this article was reported.

\begin{abstract}
Rosai-Dorfman disease is a rare histiocytic disorder, clinically characterized by massive, bilateral painless cervical lymphadenopathy with potential for extranodal manifestations. We report a 45-year-old male patient who presented with a slowly growing erythematous nodule of the left chin. The mass appeared non-vascular on computed tomography study, but ultrasonogram was suggestive of a vascular lesion. The lesion was excised with presumptive diagnosis of a hemangioma. However, histopathologic examination of the surgical biopsy revealed histiocytic infiltration with emperipolesis, which was pathognomic for Rosai-Dorfman disease. Additional imaging studies did not reveal lymph node enlargement or other extranodal manifestation. The patient was diagnosed with cutaneous form of the Rosai-Dorfman disease and was discharged home. He remains free of local recurrence at 8 months.
\end{abstract}

Keywords: Rosai-Dorfman disease / Hemangioma / Computed tomography / Ultrasonography

\section{INTRODUCTION}

Rosai-Dorfman disease is a rare histiocytic disorder initially described in 1969 by Rosai and Dorfman [1] under the term sinus histiocytosis with massive lymphadenopathy. Clinically, the disease is characterized by massive, bilateral painless cervical lymphadenopathy and can be accompanied by fever, leukocytosis with neutrophilia, elevated erythrocyte sedimentation rate and polyclonal gammopathy [2-5]. Extranodal involvement occurs in 43\% of cases with anatomic distribution that includes nasal and paranasal cavities, eyes and retro-orbital tissue, central nervous system, bones, and skin [2,4,6-10]. In this case report, we present a patient with a cutaneous form of Rosai-Dorfman disease.

Correspondence: Sung Won Jung

Department of Plastic and Reconstructive Surgery, Hallym University Sacred Heart Hospital, Hallym University Medical Center, 22 Gwanpyeong-ro, 170 beongil, Dongan-gu, Anyang 14068, Korea

E-mail: hallymjsw@gmail.com

Received May 27, 2015 / Revised August 24, 2015 / Accepted December 3, 2015

\section{CASE REPORT}

A 45-year-old male patient presented with a painless left chin mass which had been growing in size over a 2-month period leading up to the clinic visit (Fig. 1). On examination, the nodule measured 1.5 $\mathrm{cm}$ by $1 \mathrm{~cm}$. It was erythematous and was mobile without tenderness to palpation. No lymph node enlargement could be found. The patient denied systemic symptoms such as fever, malaise, and weight loss. Ultrasonogram of the lesion revealed prominent blood flow, which was suggestive of a vascular mass such as hemangioma or arteriovenous malformation (Fig. 2). On computed tomography (CT) study, however, the lesion did not appear as hypervascular as suggested by the ultrasound study (Fig. 3).

Under the presumptive diagnosis of a hemangioma, we planned for an excision of the lesion with primary indication being cosmesis. In the operating room, the chin surrounding the lesion was anesthetized with local injection and surgically prepped. The nodule was excised in an elliptical fashion without any unusual operative findings, and the wound was closed primarily. 
On H\&E staining, the lesion was found to have infiltration of large histiocytes, lymphocytes, and plasma cells (Fig. 4). Immunohistochemical stain of the specimen was positive for S-100 protein and CD 68 (Fig. 5) but negative for CDla. Final pathologic diagnosis was Rosai-Dorfman disease.

Because systemic involvement is a frequent presentation for Rosai-Dorfman, additional CT studies were performed for neck, chest, and abdomen. The studies were negative for lymph node enlargements, and no suspicious masses could be found in extranodal sites. The patient was discharged home with the diagnosis of cutaneous Rosai-Dorfman disease and remains free of local recurrence at 8 months.

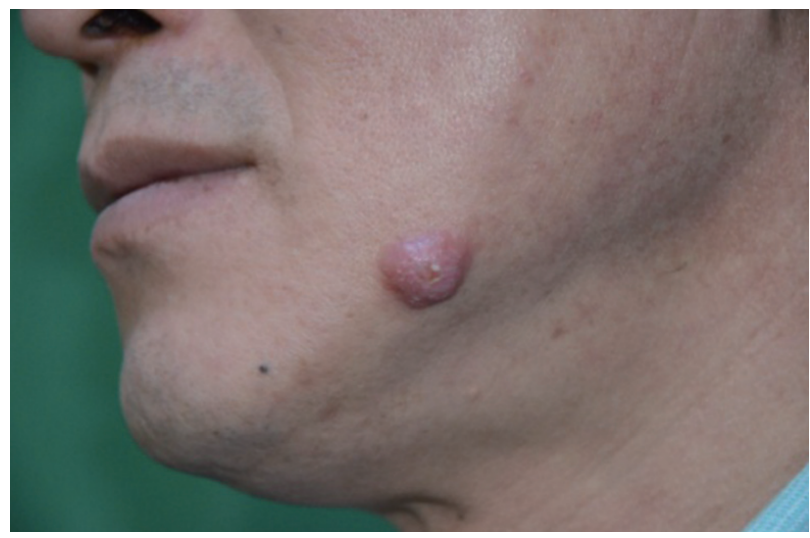

Fig. 1. This erythematous mass had grown over a 2-month period prior to presentation. The mass was soft and mobile, and was nontender to palpation.

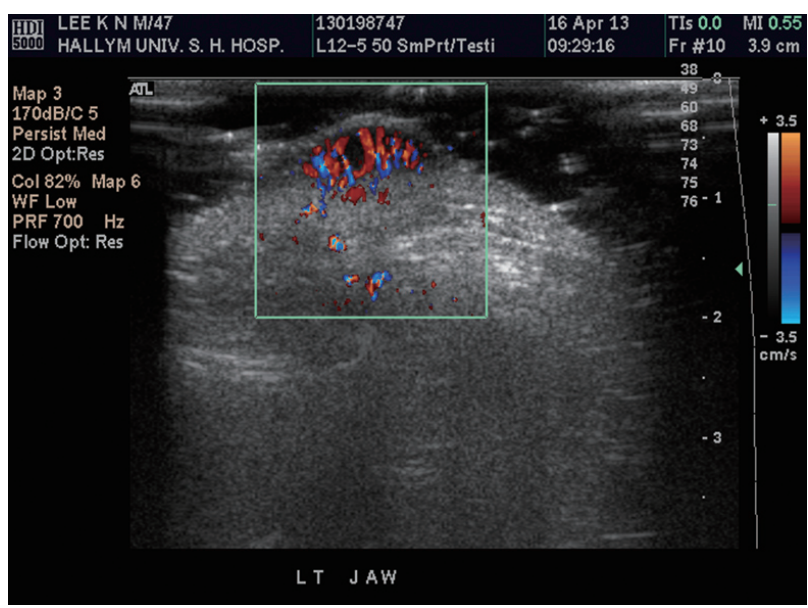

Fig. 2. Preoperative ultrasonography demonstrates prominently increased blood flow within the mass, which was suggestive of hemangioma.

\section{DISCUSSION}

The term 'cutaneous Rosai-Dorfman disease' is used for those cases in which the disease is limited to the skin. It is clinically distinct from other forms of Rosai-Dorfman disease with systemic involvement [1,11-13]. Clinical features of the cutaneous disease are varied, and can be characterized by a combination of papules, plaques or nodules and pustules, granuloma annulare-like, psoriasiform, rosacea-like, xanthelasma-like, acneiform, vasculitislike, and pseudotumor-like lesions [14].

Regardless of clinical presentation, histopathologic findings are similar in all manifestations of the Rosai-Dorfman disease. In skin biopsies, a bulk of inflammatory infiltrate consisting of lymphocytes and histiocytes could be found in the upper and mid dermis or in subcutaneous tissue. The histiocytes contain large vesicular nuclei, small nucleoli, and pale cytoplasm [15-17]. Emperipolesis is the pathognomonic finding, which is the result of histiocytes engulfing intact lymphocytes surrounded by a clear halo [18]. Histiocytes show diffuse cytoplasmic and nuclear staining for S100 and CD68 but do not stain with CDla.

Generally, cutaneous Rosai-Dorfman disease does not require treatment. However, the lesions can be treated by excision, cryotherapy, steroid, retinoid, methotrexate, and thalidomide for cos-

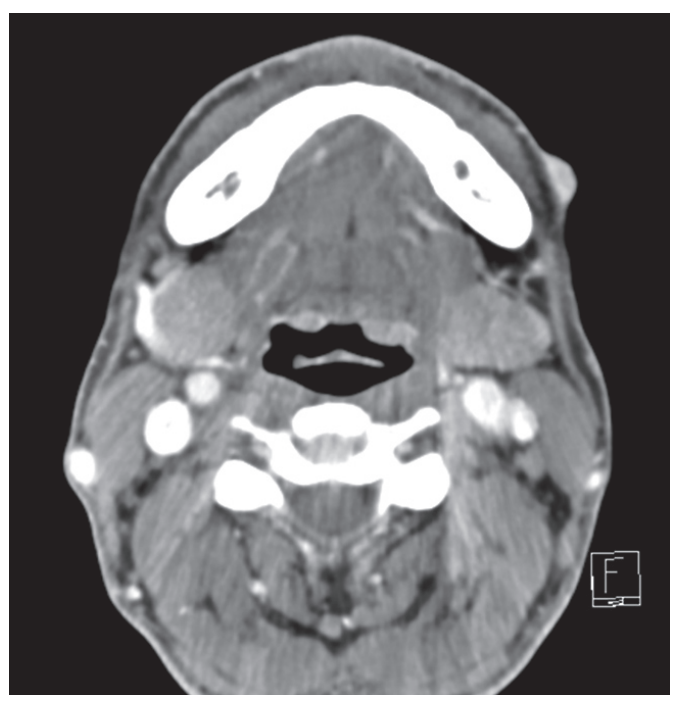

Fig. 3. Preoperative computed tomography study showed the skin nodule to be mildly enhancing, which made the hemangioma diagnosis less likely. 

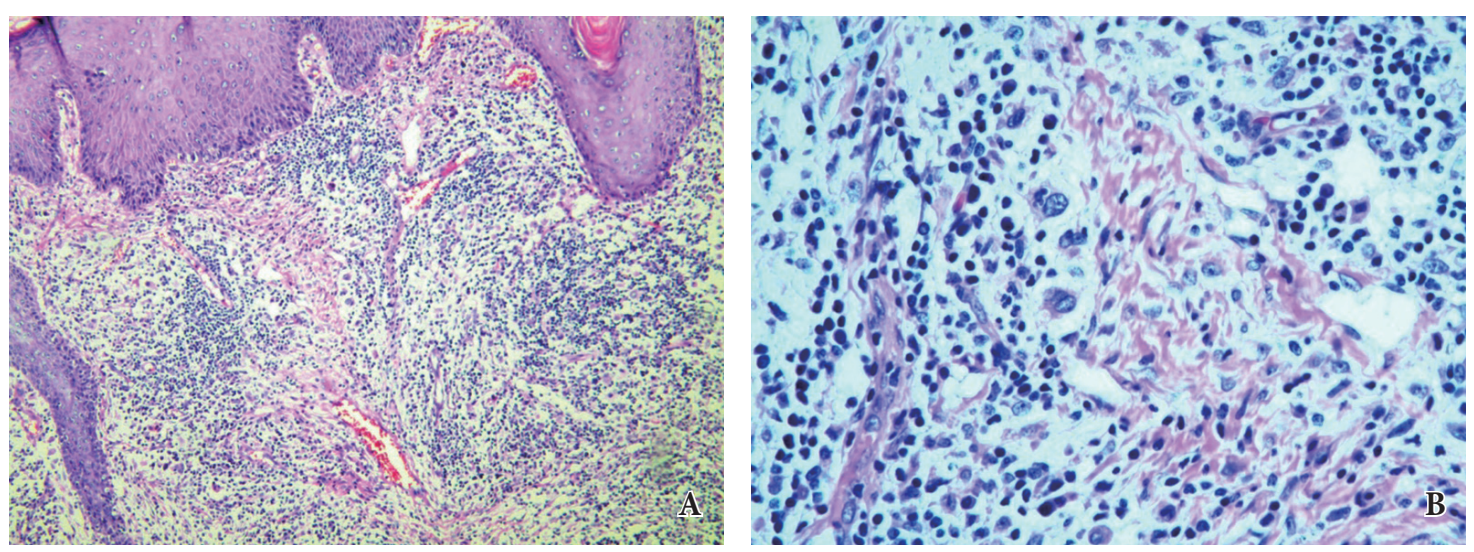

Fig. 4. (A) H\&E staining of the surgical specimen demonstrates infiltration by large histiocytes, lymphocytes, and plasma cells ( $\times 100)$. (B) A few of the histiocytes exhibit lymphophagocytosis $(\times 400)$.
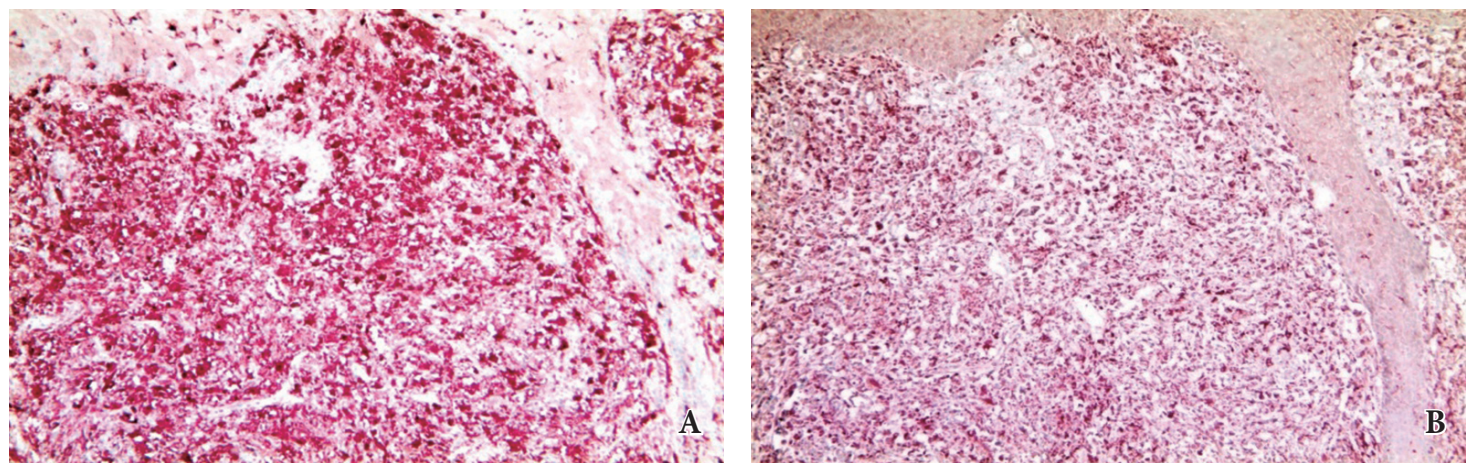

Fig. 5. Histiocytes show immunoreactivity for $S-100(A, \times 100)$ and CD68 $(B, \times 100)$.

metic indication [14].

Only a few studies have reported the use of radiologic studies such as CT, fluorodeoxyglucose positron emission tomographycomputed tomography, and magnetic resonance imaging to evaluate Rosai-Dorfman disease [19]. However, imaging studies for cutaneous form of this disease have not been reported because the disease is rare and because pathologic findings are much more important for diagnosis. In our patient, the ultrasonographic study suggested the preoperative lesion to be a hemangioma, and the lesion was excised under the presumptive diagnosis of hemangioma. If we had obtained a punch biopsy of the lesion prior to imaging and the pathologic exam had revealed the diagnosis of cutaneous Rosai-Dorfman disease, we might have chosen a treatment modality other than excision.

Cutaneous Rosai-Dorfman is a rare disease and cannot be ruled out from clinical presentation. As it was with our case, preoperative imaging studies are not helpful. Pathologic examination is required for definite diagnosis, and additional lesions must be ruled out because systemic manifestations are common.

\section{REFERENCES}

1. Rosai J, Dorfman RF. Sinus histiocytosis with massive lymphadenopathy: a newly recognized benign clinicopathological entity. Arch Pathol 1969;87:63-70.

2. Pitamber HV, Grayson W. Five cases of cutaneous Rosai-Dorfman disease. Clin Exp Dermatol 2003;28:17-21.

3. Menzel C, Hamscho N, Dobert N, Grunwald F, Kovacs AF, Wolter M, et al. PET imaging of Rosai-Dorfman disease: correlation with histopathology and ex-vivo beta-imaging. Arch Dermatol Res 2003;295:280-3.

4. Van Zander J. Cutaneous Rosai-Dorfman disease. Dermatol Online J 2004;10:12.

5. Weitzman S, Jaffe R. Uncommon histiocytic disorders: the nonLangerhans cell histiocytoses. Pediatr Blood Cancer 2005;45:256-64.

6. Salim A, Williamson M, Barker F, Hughes J. Steroid responsive cutaneous Rosai-Dorfman disease associated with uveitis and hypothyroidism. Clin Exp Dermatol 2002;27:277-9. 
7. Wang KH, Chen WY, Liu HN, Huang CC, Lee WR, Hu CH. Cutaneous Rosai-Dorfman disease: clinicopathological profiles, spectrum and evolution of 21 lesions in six patients. Br J Dermatol 2006;154:277-86.

8. Cheng SP, Jeng KS, Liu CL. Subcutaneous Rosai-Dorfman disease: is surgical excision justified? J Eur Acad Dermatol Venereol 2005;19:747-50.

9. Ortonne N, Fillet AM, Kosuge H, Bagot M, Frances C, Wechsler J. Cutaneous Destombes-Rosai-Dorfman disease: absence of detection of HHV-6 and HHV-8 in skin. J Cutan Pathol 2002;29:113-8.

10. Grabczynska SA, Toh CT, Francis N, Costello C, Bunker CB. RosaiDorfman disease complicated by autoimmune haemolytic anaemia: case report and review of a multisystem disease with cutaneous infiltrates. Br J Dermatol 2001;145:323-6.

11. Brenn T, Calonje E, Granter SR, Leonard N, Grayson W, Fletcher CD, et al. Cutaneous rosai-dorfman disease is a distinct clinical entity. Am J Dermatopathol 2002;24:385-91.

12. Hsu MY, Murphy G. Cutaneous lymphomas and leukemias. In: Elder DE, Elenitsas R, Lever WF, editors. Lever's histopathology of the skin. 10th ed. Philadelphia: Wolters Kluwer/Lippincott Williams \& Wilkins; 2009.p. 911-68.

13. Bialynicki-Birula R, Sebastian-Rusin A, Maj J, Wozniak Z, Baran E, Dziegiel P. Multicentric reticulohistiocytosis with S100 protein posi- tive staining: a case report. Acta Dermatovenerol Croat 2010;18:35-7.

14. Kutlubay Z, Bairamov O, Sevim A, Demirkesen C, Mat MC. RosaiDorfman disease: a case report with nodal and cutaneous involvement and review of the literature. Am J Dermatopathol 2014;36:353-7.

15. Lu CI, Kuo TT, Wong WR, Hong HS. Clinical and histopathologic spectrum of cutaneous Rosai-Dorfman disease in Taiwan. J Am Acad Dermatol 2004;51:931-9.

16. Shi XY, Ma DL, Fang K. Cutaneous Rosai-Dorfman disease presenting as a granulomatous rosacea-like rashs. Chin Med J (Engl) 2011;124:793-4.

17. Cangelosi JJ, Prieto VG, Ivan D. Cutaneous Rosai-Dorfman disease with increased number of eosinophils: coincidence or histologic variant? Arch Pathol Lab Med 2011;135:1597-600.

18. Kusutani N, Tamiya H, Tsuruta D, Mizuno N, Sowa J, Kaida M, et al. Apoptosis of neutrophils resulting after emperipolesis in cutaneous Rosai-Dorfman disease: a new ultrastructural finding. J Cutan Pathol 2011;38:529-31.

19. Huang JY, Lu CC, Hsiao CH, Tzen KY. FDG PET/CT findings in purely cutaneous Rosai-Dorfman disease. Clin Nucl Med 2011;36:e13-5. 